\title{
Professor Dr. Herbert Krüger 80 Jahre
}

Professor Dr. Herbert Krüger hat am 14. Dezember 1985 sein achtzigstes Lebensjahr vollendet. Seine Widersetzlichkeit gegenüber "Festgaben", selbst "Sonderheften" und ähnlichen Veranstaltungen, ist bekannt bei denen, die es angeht. Die seinerzeitigen Redakteure von "Verfassung und Recht in Ubersee", Brun-Otto Bryde und Henning von Wedel, haben darüber vor zehn Jahren an gleicher Stelle milde Klage geführt (VRU, Jahrgang 8, S. 318).

Erlaubt ist jedenfalls, hier vor der Offentlichkeit Dank zu sagen für das, was diese Zeitschrift Herbert Krüger, ihrem Begründer und Begleiter über achtzehn Jahre, auch im vergangenen Jahrzehnt verdankt: ihre Existenz. Dies gilt materiell ebenso wie immateriell - und wenn uns richtig berichtet wird, daß Maecenas die Dichter Roms nicht nur förderte, sondern ihnen auch geistige Wege wies, dann ist Herbert Krüger gewiß unser Maecenas, aber noch mehr als das: Er hat das Programm entworfen, um dessen Umsetzung sich diese Zeitschrift bemüht. Sein Rat, seine Leidenschaft für die Sache, seine Fähigkeit, Probleme aufzuspüren und namhaft zu machen, waren unentbehrlich; nur mit seiner Hilfe konnte die Zeitschrift neue Freunde und Mitarbeiter im In- und Ausland gewinnen.

So wie das zweite Lieblingskind Herbert Krügers neben seiner Zeitschrift, sein "Arbeitskreis für Überseeische Verfassungsvergleichung", in diesem Jahr nach zehn Jahren fruchtbarer Arbeit das Jubiläum ohne viel Aufhebens, durch die Tat vielmehr, beging (worüber an anderer Stelle berichtet wurde: Neue Juristische Wochenschrift 1985, S. 2880), so soll auch dieses Heft für sich selbst sprechen und damit zeigen, wie Krügers Idee, Juristen und Sozialwissenschaftler zu gemeinsamer Arbeit an den rechtlichen, institutionellen und sozialen Wandlungen in den Staaten der überseeischen Kontinente zu versammeln, heute Gestalt hat: Thematische Breite, Nähe zum politischen Geschehen und das Aufgreifen brennender Probleme hatte Herbert Krüger seinerzeit der Zeitschrift ver ordnet.

Wolfgang Graf Vitzthums dem Jubilar dedizierter Aufsatz zu Frieden und Krieg im Völkerrecht, Joachim von Stockhausens Analyse der Verschuldungskrise und ihrer Konsequenzen für die Entwicklungshilfe, Philip McNamaras Problemschau zur australischen Ureinwohner-Gesetzgebung, Petra Williams-Vedders Anmerkungen zu Rechtsfragen, die nordamerikanische Indianer betreffen, die Bestandsaufnahme des Freiherrn Klaus von der Ropp zum Krisenherd Südafrika sollen dokumentieren, daß die Redaktion auch heute versucht, Krügers Anspruch einzulösen. Hellmuth Heckers Uberblick über die Staatsangehörigkeitsbestimmungen in den Verfassungen der Welt knüpft an Arbeiten an, die schon unter der Ägide Herbert Krügers betrieben wurden, als dieser Direktor der Forschungsstelle für Völkerrecht war, der Vorgängerin des heutigen Instituts für internationale Angelegenheiten der Universität Hamburg; dieser Beitrag zeigt, daß zur 
"überseeischen Verfassungsvergleichung" in Krügers Sinne auch die Aufgabe gehört, Normen zu sichten und zusammenzuordnen, Kärrner-Arbeit also.

So ist dieses Heft gedacht als ein Versuch, dem Jubilar zu zeigen, daß die Zeitschrift auf dem von ihm gewiesenen Weg fortschreiten möchte. Auf diesem Wege wünschen wir uns weiterhin seine Ermutigung und seinen Rat.

Philip Kunig 